\title{
Stressing the stepchild: assessing right ventricular contractile reserve in pulmonary arterial hypertension
}

\author{
Brian A. Houston and Ryan J. Tedford
}

Affiliation: Division of Cardiology, Dept of Medicine, The Johns Hopkins School of Medicine, Baltimore, MD, USA.

Correspondence: Ryan J. Tedford, Section of Heart Failure, Mechanical Circulatory Support and Cardiac Transplantation, Division of Cardiology, Dept of Medicine, Johns Hopkins Medical Institutions, 568 Carnegie, 600 North Wolfe Street, Baltimore, MD 21287, USA. E-mail: Ryan.Tedfordajhmi.edu

0

@ERSpublications

Diminished right ventricular contractile reserve may improve prognostic assessment of PAH http://ow.ly/GVHsg

When Eugene Braunwald addressed the Right Heart Failure Summit in 2012, he echoed the sentiments of cardiologists throughout history by saying that he had "not paid [his] dues to the right ventricle" [1]. For years, the right ventricle was viewed as merely a conduit for transmitting blood to the lungs, a thought buttressed by the relatively preserved cardiac output seen in patients who underwent the Fontan operation [2]. In 1943, STARR et al. [3] found that venous pressure failed to augment after near-complete cauterisation of the right ventricular free wall, again supporting the concept that right ventricular function has very little effect on systemic venous pressure. What STARR et al. [3] failed to realise, but was later demonstrated by Damiano et al. [4], is that his model failed to account for the continued function of the interventricular septum, which was sufficient to propel blood through a pulmonary vascular circuit with normal resistance. Since then, it has been demonstrated inarguably that right ventricular function predicts clinical outcomes in a myriad of conditions including heart failure with preserved $[5,6]$ or reduced $[7,8]$ ejection fraction, after implantation of left ventricular assist devices [9], and pulmonary arterial hypertension (PAH) [10]. When distilling the field's rising recognition of the importance of the right ventricle, Dr Braunwald declared it a "stepchild no more".

In this issue of the European Respiratory Journal (ERJ), two studies by GuiHaire et al. [11] and Sharma et al. [12] take a novel approach to "stressing the stepchild", namely studying the use of dobutamine to assess right ventricular contractile reserve. Contractile reserve as a prognostic variable has long been the sole province of the left ventricle, predicting outcomes in valvular disease, ischaemic disease and cardiomyopathy [13-15]. The left ventricle's shape and concentric contractile pattern readily recommend it to echocardiographic evaluation of contractile function, while assessment of right ventricular contractile function by the same methods is hindered by its crescentic, triangular shape and unique longitudinal contractile pattern. Echocardiographic assessment of right ventricular contractile function often relies on imperfect one-dimensional measurements such as the tricuspid annular plane systolic excursion (TAPSE) or tricuspid annular systolic velocity $\left(S^{\prime}\right)$, or in some cases, right ventricular fractional area change (RVFAC). All of these measures, as well as right ventricular ejection fraction (which can be assessed by magnetic resonance imaging), are load dependent and thus do not represent true right ventricular contractile function $[16,17]$. The gold standard for assessing right ventricular contractility and its coupling to pulmonary arterial load necessitates the use of high-fidelity catheters to simultaneously measure pressure and volume. Constructing a family of pressure-volume loops during load alteration allows for determination of the end-systolic pressure-volume relationship, the slope of which is end-systolic elastance (Ees), a "load-independent" measure of contractility. Afterload can also be estimated on the

Received: Dec 202014 | Accepted: Dec 222014

Conflict of interest: None declared.

Copyright (CERS 2015 
pressure-volume loop as effective arterial elastance (Ea) (end-systolic pressure/stroke volume), a "lumped" parameter that accounts for both resistive and pulsatile components. The ratio of elastances $(E$ es/Ea) therefore describes the relationship between right ventricular contractility and afterload, so-called right ventricle-pulmonary artery (RV-PA) coupling [18]. In addition to the aforementioned difficulties imaging the right ventricle, a clinically apparent decline in ejection fraction or stroke volume may not occur until late in disease (i.e. Ees/Ea below 0.8-1.0). Therefore, measures of RV-PA coupling may provide the best way to detect "hidden" or borderline right ventricular function. It has been demonstrated that the "uncoupling" of right ventricular contractile function and afterload can expose subclinical right ventricular dysfunction in patients with systemic sclerosis-associated PAH [19]. GHUYSEN et al. [20] recently demonstrated that in the face of gradually rising afterload $(E a)$ induced by successive pulmonary emboli, the right ventricle can recruit contractile function (Ees) to optimise coupling only so much $\left(E_{\mathrm{es}} / E_{\mathrm{a}} \sim 1.0\right)$. At a certain increasing level of afterload, RV-PA coupling (defined by Ees/Ea) deteriorates, and the right ventricle dilates and begins to fail. The use of multibeat pressure-volume loops to assess right ventricular contractile function and RV-PA coupling is, of course, impractical in everyday clinical practice. We require a simpler, more practical and preferably noninvasive tool to measure RV-PA coupling for accurate prognostication. Single-beat methods to calculate right ventricular $E_{\text {es }}$ and $E$ es/Ea have been proposed but rely on assumptions that may not hold true under pathological conditions and require further validation [21-23]. Taken in combination, the studies by GuiHaire et al. [11] and Sharma et al. [12] argue that assessment of right ventricular contractile reserve may provide such a tool.

GuiHaire et al. [11] performed right ventricular pressure-volume analysis in a pig model of chronic pressure overload pulmonary hypertension. The study subjected 13 pigs to left pulmonary artery ligation followed by weekly injections of $n$-butyl-2-cyanoacrylate into the segmental arteries over a period of 5 weeks. Six additional pigs underwent a sham surgery that included midline sternotomy. The authors employed haemodynamic (change in stroke volume index (SVI), change in maximal rate of rise of right ventricular pressure $\left(\mathrm{d} P / \mathrm{d} t_{\max }\right)$ and change in $\left.E \mathrm{es}\right)$ measurements to assess contractile reserve. At 6 weeks, the intervention group predictably developed PAH at rest with higher right-sided filling pressures, and right ventricular dysfunction and dilation when compared with sham controls (by echocardiography). While contractility was increased in the PAH animals at rest (higher Ees and $\mathrm{d} P / \mathrm{d} t \max$ ), there was evidence of RV-PA uncoupling with significantly reduced Ees/Ea. With stress, the sham group demonstrated greater relative increases in all haemodynamic measurements of contractility and maintained RV-PA coupling. The authors found that $\Delta$ SVI and $\Delta \mathrm{d} P / \mathrm{d} t$ max were strongly correlated with impaired RV-PA coupling at rest in a linear fashion. Although $\Delta E$ es with stress was only moderately associated with resting RV-PA coupling, there was a markedly lower average $\Delta E$ es in the pulmonary hypertension group compared with the sham group. RVFAC at rest was more strongly associated with all haemodynamic measures of right ventricular contractile reserve than were right ventricular myocardial performance index and TAPSE.

In short, GUIHAIRE et al. [11] show that the absence of right ventricular contractile reserve is correlated with RV-PA uncoupling at rest in an animal model of PAH, a novel and important finding. However, this study leaves several important questions unanswered. The model of PAH employed may differ from the human condition in crucial ways. The injection of $n$-butyl-2-cyanoacrylate could conceivably have a deleterious effect on left ventricular function, and indeed left ventricular ejection fraction (LVEF) was significantly reduced in the animals with pulmonary hypertension. Though not specifically mentioned, the reduced LVEF in PAH animals in the presence of similar stroke volumes in both groups may suggest that PAH animals had a more dilated left ventricle, which is different from the small, compressed left ventricle seen in many PAH patients. This might be attributed to the pericardiectomy performed on the experimental animals, which itself may introduce a physiologically significant alteration from human PAH pathophysiology. As the authors note, RAINA et al. [24] recently demonstrated that the right ventricle markedly alters its contractile pattern after pericardiectomy, with the predominance of contractile function shifting from longitudinal (approximated by TAPSE) to transverse (more closely approximated by RVFAC). Thus, the correlation in the current study seen between RVFAC at rest and contractile reserve carries the caveat of the concomitant absence of pericardium, a condition not met by many PAH patients. However, the data are in keeping with other work that has suggested longitudinal shortening may be less predictive of clinical worsening than changes in transverse motion [25]. Future studies correlating echocardiographic parameters of contractile reserve and RV-PA coupling should carefully consider the role of the pericardium (which, similar to the right ventricle's historical perception, has been long-relegated to the status of "minimally physiologic" in normal states). In addition, one must remember that $\mathrm{d} P / \mathrm{d} t \max$ and SVI are not pure measures of right ventricular contractile reserve as they are both load dependent [26]. As pointed out, analysis of preload-recruitable stroke work [27] might prove to be superior measures of pure right ventricular contractile reserve. The authors' assertion that the use of low-dose dobutamine may have limited ability to increase stroke volume in patients with $\mathrm{PAH}$ is intriguing, but requires further study in light of the left ventricular changes that occurred in their model. Finally, it would have been enlightening 
to further study echocardiographic markers of right ventricular contractile reserve in search of a noninvasive correlative parameter to the haemodynamic markers of contractile reserve and RV-PA coupling.

The study by SHARMA et al. [12] may help answer this final question. The authors compared right ventricular contractile reserve during dobutamine stress (defined by the echocardiographic parameters $S^{\prime}$ and TAPSE) in patients with PAH compared with age-matched controls. A subset of PAH patients then underwent cardiopulmonary exercise testing. The underlying aetiology was largely a mix of idiopathic and scleroderma-related PAH. Most of the PAH patients were in New York Heart Association functional class II-III functional class (13 out of 16 patients) and were on at least one pharmacological agent for treatment of PAH. The PAH group had a significantly reduced TAPSE and $S^{\prime}$ at rest and demonstrated markedly attenuated contractile reserve, as measured by TAPSE and $S^{\prime}$, compared with controls. Intriguingly, there was a subset of PAH patients with relatively well preserved TAPSE (10 out of 16 had TAPSE $\geqslant 1.6 \mathrm{~cm})$ and $S^{\prime}$ (seven out of 16 had $S^{\prime} \geqslant 10 \mathrm{~cm} \cdot \mathrm{s}^{-1}$ ) at rest. Both of these patient subgroups demonstrated depressed contractile reserve when compared with controls, whether assessed by TAPSE or $S^{\prime}$. 13 PAH patients subsequently underwent cardiopulmonary exercise testing. Mean peak oxygen consumption $\left(V^{\prime} \mathrm{O}_{2}\right)$ and exercise capacity were reduced, and demonstrated a significant positive correlation with $\Delta S^{\prime}$. Resting TAPSE and $S^{\prime}$ were less strongly associated with peak $V^{\prime} \mathrm{O}_{2}$.

When considering this study, several important questions arise. As noted earlier, patients with idiopathic $\mathrm{PAH}$ and systemic sclerosis-related PAH have intrinsically different diseases. In addition to worse RV-PA coupling $[19,28]$, musculoskeletal limitations and lung comorbidities may also affect exercise capacity in systemic sclerosis [29]. The inclusion of both patient types in the current study might serve to obfuscate results reliant on right ventricular contractile reserve, and future studies should account for the heterogeneity of these disease types, particularly when it comes to the right ventricle. Likewise, the assessment of other echocardiographic parameters (RVFAC) might have provided superior discernment of impaired right ventricular contractile reserve. As noted earlier, GuiHAire et al. [11] found RVFAC to have a stronger correlation than TAPSE with haemodynamic assessment of contractile reserve. Future studies should also consider assessment of right ventricular strain as a marker of right ventricular contractile reserve. In a previous issue of the ERJ, LAMIA et al. [30] demonstrated that PAH patients had a global reduction in right ventricular longitudinal strain and that even in patients with preserved TAPSE, speckle tracking strain imaging showed right ventricular dyssynchrony.

Despite these concerns, the finding that right ventricular contractile reserve, as assessed by a reproducible, noninvasive echocardiographic parameter such as $S^{\prime}$, is predictive of exercise capacity is important. Perhaps most provocative is the discovery that even patients with normal resting parameters of right ventricular function demonstrated impaired contractile reserve, which correlated with their exercise capacity and peak $V^{\prime} \mathrm{O}_{2}$. This suggests that assessment of right ventricular contractile reserve may provide superior prognostic information to resting echocardiography by unmasking intrinsic right ventricular dysfunction even in patients with normal resting findings. Recently, GRÜNIG et al. [31] employed echocardiography to assess right ventricular contractile reserve during exercise (assessed by their ability to augment pulmonary artery systolic pressure), and found that contractile reserve not only correlated with exercise capacity and peak $V^{\prime} \mathrm{O}_{2}$ but also with short- and long-term survival.

A test that accurately, reproducibly and noninvasively identifies intrinsic right ventricular contractile dysfunction in PAH patients, and specifically impaired RV-PA coupling, represents the holy grail of prognostication in PAH. Such a test would allow for improved therapeutic targeting, potentially identifying the patients who would benefit from more aggressive multidrug approaches or consideration of advanced therapies such as transplant. The two studies in this issue of ERJ represent progress toward that holy grail and the authors should be congratulated for their work. By demonstrating that impaired resting RV-PA coupling is correlated with diminished haemodynamic right ventricular contractile reserve [11] and that echocardiographic evidence of diminished right ventricular contractile reserve is correlated with poor exercise capacity, even in patients who appear normal on resting echocardiography [12], these two studies provide a blueprint for future investigations into the development of the ideal prognostic test. Future studies should investigate whether the echocardiographic parameters of diminished right ventricular contractile reserve identified by SHARMA et al. [12] correlate with clinical outcomes including response to therapy and mortality. Identifying patients with normal resting echocardiographic measures of right ventricular function and stratifying them by presence of right ventricular contractile reserve to compare clinical outcomes would be of particular interest. Ultimately, investigators may explore the utility of employing the presence or absence of diminished right ventricular contractile reserve to ascribe patients to different treatment strategies. These two studies represent a large step forward in the assessment of the "stepchild ventricle". 


\section{References}

1 Braunwald E. The right ventricle: a step-child no longer. http://pvri.info/content/right-ventricle-step-child-nolonger\#.VJENMlok_ww Date last updated: March 10, 2013.

2 Fontan F, Baudet E. Surgical repair of tricuspid atresia. Thorax 1971; 26: 240-258.

3 Starr I, Jeffers WA, Meade RH. The absence of conspicuous increments of venous pressure after severe damage to the right ventricle of the dog, with discussion of the relation between clinical congestive failure and heart disease. Am Heart J 1943; 26: 291.

4 Damiano RJ Jr, La Follette P Jr, Cox JL, et al. Significant left ventricular contribution to right ventricular systolic function. Am J Physiol 1991; 261: H1514-H1524.

5 Melenovsky V, Hwang S, Lin G, et al. Right heart dysfunction in heart failure with preserved ejection fraction. Eur Heart J 2014; 35: 3452-3462.

6 Mohammed SF, Hussain I, Abou Ezzeddine OF, et al. Right ventricular function in heart failure with preserved ejection fraction: a community based study. Circulation 2014; 130: 2310-2320.

7 Ghio S, Gavazzi A, Campana C, et al. Independent and additive prognostic value of right ventricular systolic function and pulmonary artery pressure in patients with chronic heart failure. J Am Coll Cardiol 2001; 37: $183-188$

8 Guazzi M, Bandera F, Pelissero G, et al. Tricuspid annular plane systolic excursion and pulmonary arterial systolic pressure relationship in heart failure: an index of right ventricular contractile function and prognosis. Am J Physiol Heart Circ Physiol 2013; 305: H1373-H1381.

9 Kirklin JK, Naftel DC, Pagani FD, et al. Sixth INTERMACS annual report: A 10,000-patient database. J Heart Lung Transplant 2014; 33: 555-564.

10 Forfia PR, Fisher MR, Mathai SC, et al. Tricuspid annular displacement predicts survival in pulmonary hypertension. Am J Respir Crit Care Med 2006; 174: 1034-1041.

11 Guihaire J, Haddad F, Noly PE, et al. Right ventricular reserve in a piglet model of chronic pulmonary hypertension. Eur Respir J 2015; 45: 709-717.

12 Sharma T, Lau EMT, Choudhary P, et al. Dobutamine stress for evaluation of right ventricular reserve in pulmonary hypertension. Eur Respir J 2015; 45: 700-708.

13 Naqvi TZ, Goel RK, Forrester JS, et al. Myocardial contractile reserve on dobutamine echocardiography predicts late spontaneous improvement in cardiac function in patients with recent onset idiopathic dilated cardiomyopathy. J Am Coll Cardiol 1999; 34: 1537-1544.

14 Quere JP, Monin JL, Levy F, et al. Influence of preoperative left ventricular contractile reserve on postoperative ejection fraction in low-gradient aortic stenosis. Circulation 2006; 113: 1738-1744.

15 Chaudhry F, Tauke J, Alessandrini R, et al. Prognostic implications of myocardial contractile reserve in patients with coronary artery disease and left ventricular dysfunction. J Am Coll Cardiol 1999; 34: 730-738.

16 Ghio S, Klersy C, Magrini G, et al. Prognostic relevance of the echocardiographic assessment of right ventricular function in patients with idiopathic pulmonary arterial hypertension. Int J Cardiol 2010; 140: 272-278.

17 Howard LS, Grapsa J, Dawson D, et al. Echocardiographic assessment of pulmonary hypertension: standard operating procedure. Eur Respir Rev 2012; 21: 239-248.

18 Champion HC, Michelakis ED, Hassoun PM. Comprehensive invasive and noninvasive approach to the right ventricle-pulmonary circulation unit: state of the art and clinical and research implications. Circulation 2009; 120: 992-1007.

19 Tedford RJ, Mudd JO, Girgis RE, et al. Right ventricular dysfunction in systemic sclerosis associated pulmonary arterial hypertension. Circ Heart Fail 2013; 6: 953-963.

20 Ghuysen A, Lambermont B, Kolh $\mathrm{P}$, et al. Alteration of right ventricular-pulmonary vascular coupling in a porcine model of progressive pressure overloading. Shock 2008; 29: 197-204.

21 Brimioulle S, Wauthy P, Ewalenko P, et al. Single-beat estimation of right ventricular end-systolic pressure-volume relationship. Am J Physiol Heart Circulatory Physiol 2003; 284: H1625-H1630.

22 Lambermont B, Segers P, Ghuysen A, et al. Comparison between single-beat and multiple-beat methods for estimation of right ventricular contractility. Crit Care Med 2004; 32: 1886-1890.

23 Vanderpool RR, Pinsky MR, Naeije R, et al. RV-pulmonary arterial coupling predicts outcome in patients referred for pulmonary hypertension. Heart 2015; 101: 37-43.

24 Raina A, Vaidya A, Gertz ZM, et al. Marked changes in right ventricular contractile pattern after cardiothoracic surgery: implications for post-surgical assessment of right ventricular function. J Heart Lung Transplant 2013; 32: 777-783.

25 Mauritz GJ, Kind T, Marcus JT, et al. Progressive changes in right ventricular geometric shortening and long-term survival in pulmonary arterial hypertension. Chest 2012; 141: 935-943.

26 Schmidt HD, Hoppe H. Preload dependence of $\mathrm{dP} / \mathrm{dt} \max$, VCE max and calculated V max compared to the inotropic sensitivity of these indices of cardiac contractility. Basic Res Cardiol 1978; 73: 380-393.

27 Feneley MP, Skelton TN, Kisslo KB, et al. Comparison of preload recruitable stroke work, end-systolic pressure-volume and $\mathrm{dP} / \mathrm{dtmax}$-end-diastolic volume relations as indexes of left ventricular contractile performance in patients undergoing routine cardiac catheterization. J Am Coll Cardiol 1992; 19: 1522-1530.

28 Fox BD, Shimony A, Langleben D, et al. High prevalence of occult left heart disease in scleroderma-pulmonary hypertension. Eur Respir J 2013; 42: 1083-1091.

29 Denton CP, Avouac J, Behrens F, et al. Systemic sclerosis-associated pulmonary hypertension: why disease-specific composite endpoints are needed. Arthritis Res Ther 2011; 13: 114.

30 Lamia B, Molano L, Viacroze C, et al. Right ventricular dyssynchrony measured using speckle tracking strain as a predictive factor in pulmonary hypertension patients. Eur Respir J 2011; 38: 1512.

31 Grünig E, Tiede H, Enyimayew EO, et al. Assessment and prognostic relevance of right ventricular contractile reserve in patients with severe pulmonary hypertension. Circulation 2013; 128: 2005-2115. 